\title{
Effects of Wolbachia-targeted tetracycline on a host-parasitoid-symbiont interaction
}

\author{
Hosagavi P. PUTTARAJU and Bandekodigenahalli M. PRAKASH \\ Laboratory of Seribiotechnology, Department of Sericulture, Jnanabharathi Campus, Bangalore University, Bangalore 560056, \\ India; e-mail: puttarajuhp@hotmail.com
}

Key words. Wolbachia, Exorista sorbillans, Tachinidae, Bombyx mori, tetracycline, fecundity, cytoplasmic incompatibility, commercial characters

\begin{abstract}
The uzifly, Exorista sorbillans (Diptera: Tachinidae), a parasite of the silkworm, Bombyx mori L. (Lepidoptera: Bombycidae), causes heavy losses to the silk industry. This parasitoid harbours a Wolbachia endosymbiont, which controls the fly's reproduction. In the present study a method for curtailing this notorious pest by administering Wolbachia-targeted tetracycline via its silkworm host's diet is investigated. Tetracycline not only influenced the larval growth of the silkworms' by decreasing larval duration, increased silk production and fecundity, without affecting hatchability, it also decreased the reproductive fitness of the uzifly endoparasite by killing the Wolbachia. The antibiotic exerts a beneficial influence by affecting the intestinal flora of silkworm larvae. On the other hand the reproductive fitness of uzifly was greatly reduced in terms of different reproductive abnormalities. When male and female flies that emerged from treated host silkworms were crossed and males from untreated hosts and females from treated hosts were crossed, approximately $72 \%$ and $97 \%$ of the eggs failed to hatch, respectively. However, of the eggs from crosses between male and female flies that emerged from untreated hosts and between males from treated hosts with females from untreated hosts, an average of 30\% failed to hatch and the Wolbachia infection enhanced the fecundity of uziflies. These results demonstrate that the Wolbachia may be essential for uzifly reproduction and that Wolbachia-targeted antibiotics have a beneficial influence on silkworm growth while decreasing the reproductive fitness of the uzifly, E. sorbillans.
\end{abstract}

\section{INTRODUCTION}

Wolbachia forms a group of endocellular, heritable, gram-negative alpha-proteobacteria that infect 16-76\% (Werren et al., 1995; Jeyaprakash \& Hoy, 2000) of all insect species. These bacteria are also found in non-insect invertebrates including nematodes (Kozek, 1997), mites (Vala et al., 2003) and spider mites (Breeuwer, 1997). A decade of research has shown that much of the success of Wolbachia can be attributed to the diverse host phenotypic effects associated with infection. These effects range from classical mutualism to reproductive parasitism as Wolbachia induces a diverse array of reproductive abnormalities in its hosts, including cytoplasmic incompatibility (Barr, 1980; Breeuwer et al., 1992; O'Neill et al., 1992), parthenogenesis (Stouthamer et al., 1993), male killing (Hurst et al., 1999), feminization of genetic males in isopods (Rousset et al., 1992), affects sperm competition (Wade \& Chang, 1995) and overrides chromosomal sex determination (Werren \& Beukeboom, 1998), as well as affecting the ageing of Drosophila simulans (Min \& Benzer, 1997). The presence of a particular group of Wolbachia is known to increase the fecundity of the parasitic wasp Asobara tabida, which shows that Wolbachia can affect reproduction in wasps (Dedeine et al., 2001, 2004). The unique biology of Wolbachia has attracted researchers interested in subjects ranging from the evolutionary implication of infections to the use of this agent for pest and disease control (Zabalou et al., 2004). In addition, the discovery of an obligate mutualistic relationship between Wolbachia and filarial nema- todes has provided a promising avenue for curing filariasis by administering infected patients with antibiotics such as tetracycline (Taylor \& Hoerauf, 2001). The elimination of Wolbachia by tetracycline treatment decreases fitness in filarial nematodes in several nonspecific ways, including survival, developmental success and reproduction (Hoerauf et al., 1999; Langworthy et al., 2000). The removal of Wolbachia using tetracycline cause infertility in females in species of Aedes (Trpis et al., 1981; Kambhampati et al., 1993).

The uzifly, Exorista sorbillans (Diptera: Tachinidae), an endoparasite of the silkworm, Bombyx mori L. (Lepidoptera: Bombycidae), causes considerable losses to the sericultural industry, which has stimulated researchers to study its biology and control measures (Channabasavanna et al., 1993). The fly is known to harbour Wolbachia (Madhu \& Puttaraju, 2001; Puttaraju \& Madhu, 2002) and the molecular genetic studies of Wolbachia in five populations of $E$. sorbillans using $16 \mathrm{~S}$ rDNA and ftsZ gene analysis showed that the bacteria belongs to the $\mathrm{B}$ clade of Wolbachia (Chatterjee et al., 2003). This bacteria-fly relationship may provide a means of curtailing this notorious pest. In the present study attempts to suppress the reproductive fitness of the uzifly while improving the growth rate of its host, through treating the host silkworm with tetracycline, were undertaken.

\section{MATERIAL AND METHODS}

\section{Silkworm collection and rearing}

Young larvae of Bombyx mori were brought from a rearing center near Bangalore, India, and reared to third instar using the 
standard silkworm mass rearing technique of Ullal \& Narasimhanna (1994). After the third instar, the larvae were allocated to one of four replicates, with 60 larvae in each replicate. In all four replicates, mulberry leaves soaked in $0.1 \mathrm{mg} / \mathrm{ml}$ oxytetracycline were fed to the larvae. A control batch was also reared on mulberry leaves soaked in distilled water. Larval growth, in terms of body weight, larval duration, cocoon weight, cocoon shell weight and silk filament length, was calculated following standard methods (Mahadevappa et al., 2000). For determining the reproductive fitness of the Pure Mysore (PM) strain of 250 larvae, a polyvoltine race, were maintained in both experimental and control conditions. The fecundity and the hatchability of their eggs were calculated by counting the number of eggshells after hatching. The silkworm larvae were reared in a cellular rearing method.

\section{Uzifly collection and rearing}

Since the main aim of the present study is to suppress the uzifly populations infesting silkworm cocoons, more than 500 silkworm larvae were mass reared and feed a diet containing 0.1 $\mathrm{mg} / \mathrm{ml}$ oxy-tetracycline. The treatment started in the second larval instar. On reaching the fourth day of the fifth instar, the silkworm larvae were exposed to parasitization by uziflies. Silkworm larvae with one to two uzifly eggs on their bodies were mass reared in wire mesh cages, $14 \times 14 \times 14$ inches (Manjunatha $\&$ Puttaraju, 1993). The mature uzifly larvae that emerged from the silkworm were collected and placed in Petri dishes containing sand, which were kept in cages until the adults emerged. The adult flies were sexed based on the genitalia and other sex specific morphological characters and provided with $8 \%$ glucose as food. The time of emergence of the uziflies was also recorded.

\section{Isolation of genomic DNA from uzifly and silkmoth}

DNA from at least one uzifly and silkmoth was extracted following the usual stepwise methods of extraction with phenol, phenol: chloroform: isoamylalcohol, chloroform and finally precipitated with two volumes of double distilled alcohol in the presence of 3M sodium acetate (Sambrook et al., 1989). The precipitated DNA was washed in $70 \%$ alcohol, dried and dissolved in $2 \mathrm{ml}$ of TE (Tris-EDTA) buffer. The DNA was subjected to RNAse-A treatments followed by further re-extraction with phenol: chloroform: isoamyl alcohol and chloroform and finally, RNA free DNA was precipitated with alcohol in the presence of $3 \mathrm{M}$ sodium acetate, the pellet was dried and dissolved in TE buffer. The concentration of $10-12 \mathrm{ng} / \mu \mathrm{l}$ was realized through quantification on $0.8 \%$ agarose gel (Gibco BRL, USA) using standard concentration of lambda DNA in TrisBoric acid-EDTA (ethylenediaminetetra-acetic acid) buffer.

\section{PCR-amplification of uzifly and silkmoth DNA for use in detecting Wolbachia}

A polymerase chain reaction assay based on specific amplification of the Wolbachia-wsp gene primer pair wsp81F 5'- TGG TCC AAT AAG TGA TGA AGA AAC - 3' and wsp691R 5'AAA AAT TAA ACG CTA CTC CA - 3' was used to detect Wolbachia in individual flies/moth using a PTC 200 of MJ Research Thermocycler, and a $20 \mu 1$ reaction mixture containing $1 \times$ PCR buffer, $0.2 \mathrm{mM}$ dNTP's, $2.5 \mathrm{mM} \mathrm{MgCl}_{2}$, and $0.5 \mathrm{U}$ Taq DNA Polymerase (MBI-Fermentas, USA), $0.1 \mu \mathrm{M}$ of each forward and reverse primers (Bangalore Genei), $50 \mathrm{ng}$ template DNA and final volume of millipore water to make up $20 \mu$ l. The PCR was done using an initial denaturation step at $94^{\circ} \mathrm{C}$ for 2 min followed by 40 cycles with denaturation step at $94^{\circ} \mathrm{C}$ for 1 min, primer annealing at $55^{\circ} \mathrm{C}$ for $2 \mathrm{~min}$ and primer extension with the presence of Taq-DNA polymerase at $72^{\circ} \mathrm{C}$ for $2 \mathrm{~min}$ and final extension at $72^{\circ} \mathrm{C}$ for $5 \mathrm{~min}$. The amplified PCR prod- ucts were separated through $1.3 \%$ agarose gel electrophoresis run in $1 \times$ TBE (Tris- Boric acid- EDTA) buffer for a length of $5-6 \mathrm{~cm}$ at a constant $65 \mathrm{~V}$ from a Pharmacia power supply EPS $200 \mathrm{~V} / 400 \mathrm{~mA}$. The gel was stained with $0.5 \mu \mathrm{g} / \mathrm{ml}$ of gel with ethidium bromide just prior to casting. The documentation was done using gel documentation system in Genesnap software version 5.0.

\section{Crossing of silk moths}

Immediately after emergence silk moths were crossed in the following order:

1. Untreated males $\times$ untreated females.

2. Untreated males $\times$ treated females.

3. Treated males $\times$ treated females.

4. Treated males $\times$ untreated females.

There were 20 replicates of each cross, which occurred in ring-cellules of $5 \mathrm{~cm}$ diameter for a period of $4 \mathrm{~h}$ followed by urination for $10 \mathrm{~min}$, after which they oviposited for $30 \mathrm{~h}$ on cardboard paper in semi-dark condition. The eggs were washed in distilled water and incubated at $26 \pm 1^{\circ} \mathrm{C}$. After hatching, the number of hatched and infertile eggs were recorded.

\section{Crossing of uziflies}

One day after emergence, crosses were performed in the following order by containing two males (to ensure mating) with one female, replicated ten times:

1. Males $\times$ females, both emerged from untreated hosts.

2. Males $\times$ females, both emerged from treated hosts.

3. Males from treated hosts $\times$ females untreated hosts.

4. Males from untreated hosts $\times$ females from treated hosts.

After mating for a day the flies were provided with 25 early $5^{\text {th }}$ instar silkworm larvae for oviposition. The silkworm larvae were replaced every $12 \mathrm{~h}$ for a period of five days. Fecundity and hatchability were calculated by counting the eggs and the black scars, which appeared after hatching, respectively, on the bodies of silkworm larvae. The larvae were reared in the laboratory in the cellular rearing unit for further observations. The data were analyzed using Students' t-test for paired samples and one-way ANOVA for multiple samples (Khan \& Khanum, 1994).

\section{RESULTS}

\section{Infection status of Wolbachia in silkworms and uziflies}

All the control male and female uziflies from laboratory and field population tested positive (Fig. 1) for Wolba-

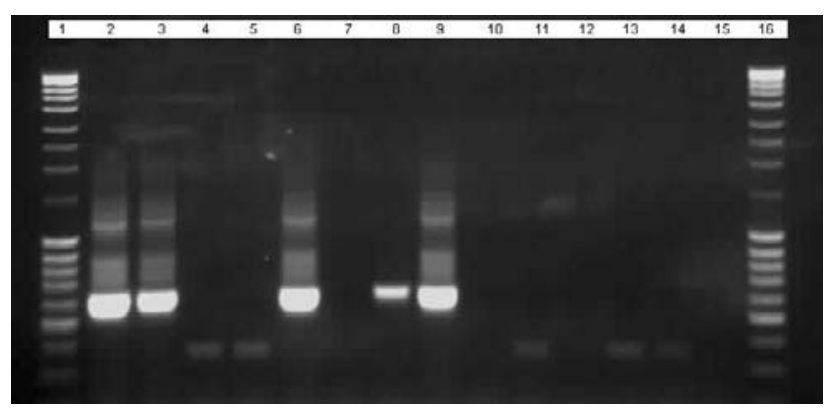

Fig. 1. PCR product of uziflies and silkworm DNA. Lane 1 and 16 - Mass ruler (MBI-Fermentas); Lane 2 and 3 - control populations from field and laboratory; Lane 4 and 5 - direct tetracycline treated uziflies and uziflies that emerged from tetracycline treated silkworms; Lane 6-duplicate laboratory population; Lane 7 - temperature treated population; Lane 8 and 9 - two and three week old uziflies; Lane 10-15 - different races of silkworm. 


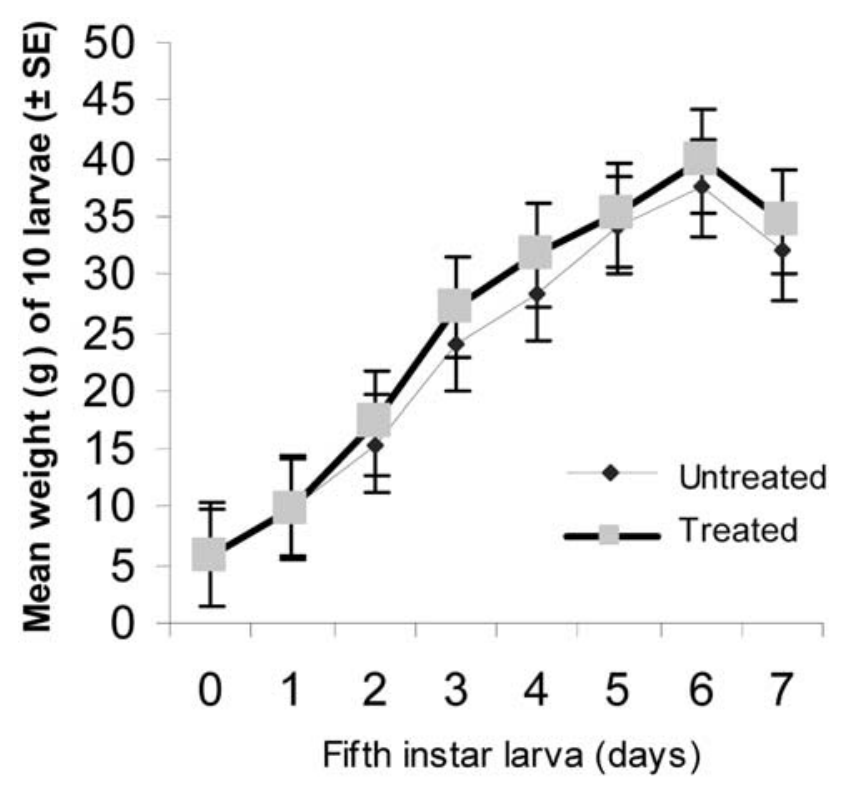

Fig. 2. Weight of treated and untreated larvae.

chia wsp (Wolbachia surface protein) gene, which consists of around $600 \mathrm{bp}$, and the treated male and female uziflies and all silkworm races tested negative, which is in accord with our earlier findings (Madhu \& Puttaraju, 2001; Puttaraju \& Madhu, 2002) and suggests a high prevalence of Wolbachia in uziflies.

\section{Effect of tetracycline on silk productivity by silkworm}

This study revealed that oral administration of the antibiotic oxy-tetracycline stimulated the growth, development and silk production of silkworm, Bombyx mori. L. The data given in Fig. 2 reveals that tetracycline, when administered orally along with mulberry leaves, resulted

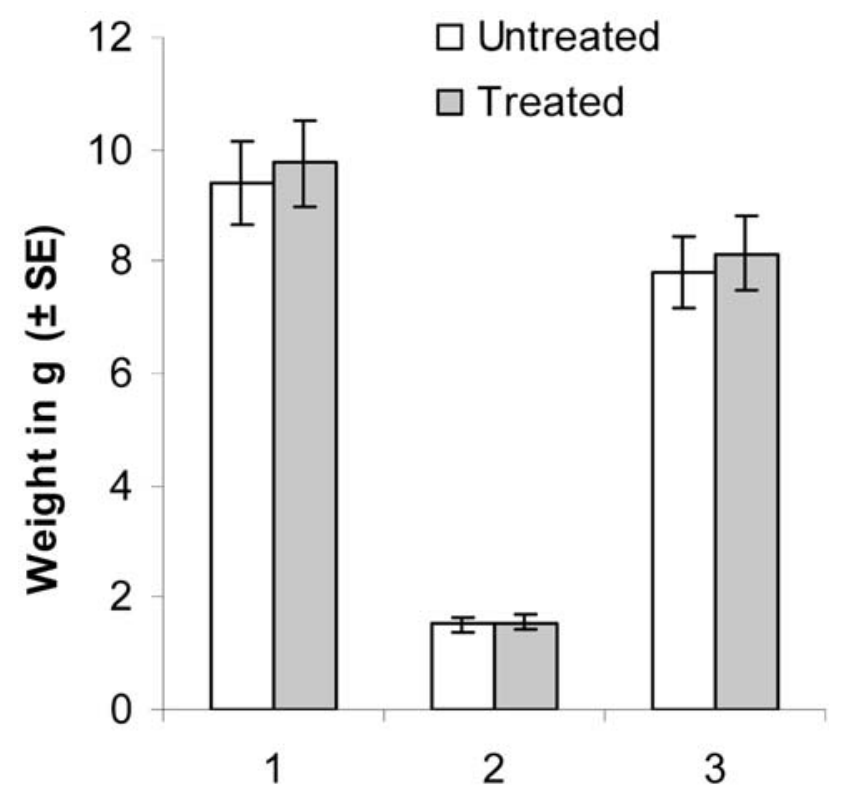

Fig. 3. Average weight of cocoon plus pupae, silken cocoon and pupae produced by treated and untreated silkworm larvae. 1 - cocoon plus pupal weight; 2 - silken cocoon weight; 3 - pupal weight.

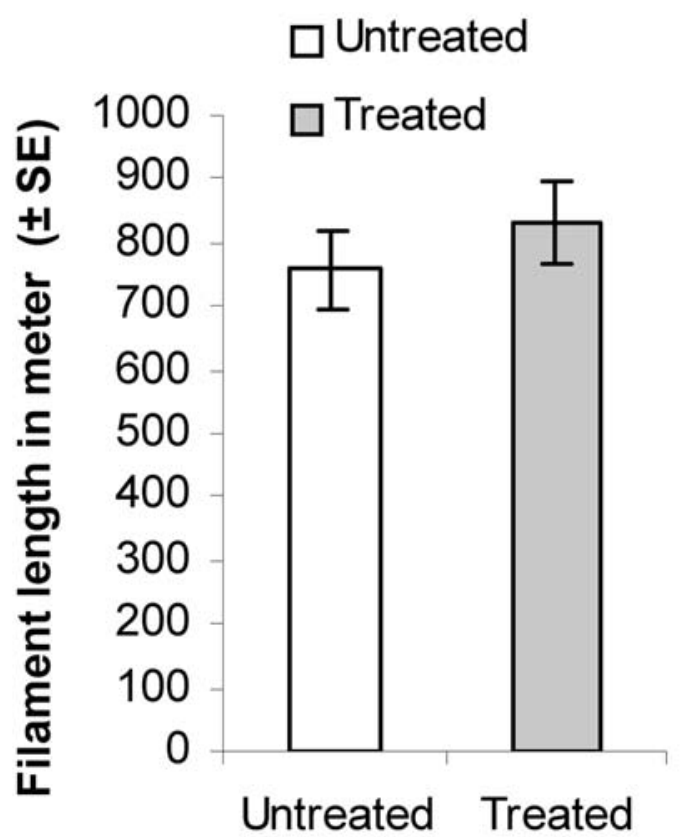

Fig. 4. Silk filament length produced by untreated and treated silkworm larvae.

in a significant increase in the body weight of the larvae. Untreated six day-old final instar larvae weigh $37.463 \pm$ $0.35 \mathrm{gm}(\mathrm{n}=4)$ and the tetracycline-treated larvae of the same age weigh $39.8 \pm 0.29 \mathrm{gm}(\mathrm{n}=4)$ (the values are the means of 10 larvae \pm SE) $(\mathrm{t}=5.09$, d.f. $=6, \mathrm{p}<0.01)$. However, the duration of development of the treated larvae was 18 to $20 \mathrm{~h}$ less than that of untreated larvae. More importantly the cocoon weights of the treated silkworms were significantly greater than those of the controls: $9.399 \pm 0.063 \mathrm{gm}(\mathrm{n}=7)$ for the untreated larvae and $9.745 \pm 0.052 \mathrm{gm}(\mathrm{n}=8)$ for the tetracycline-treated larvae (the values are the means of 5 cocoons $\pm S E ; t=$

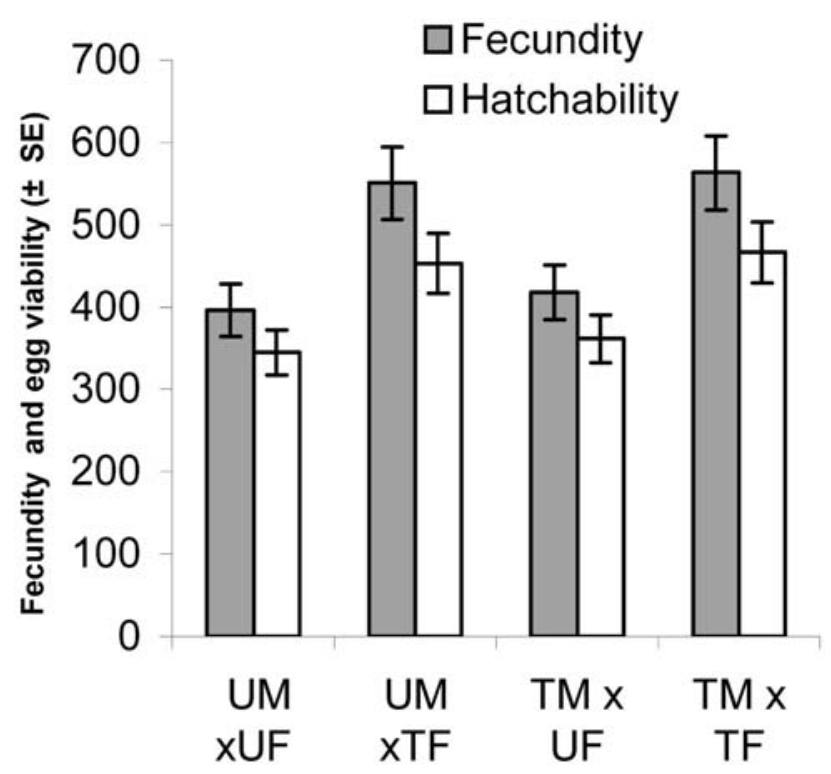

Fig. 5. Fecundity and egg viability of treated and untreated silkworms. $\mathrm{U}$ - untreated; $\mathrm{T}$ - treated; $\mathrm{M}$ - male; F - female. 


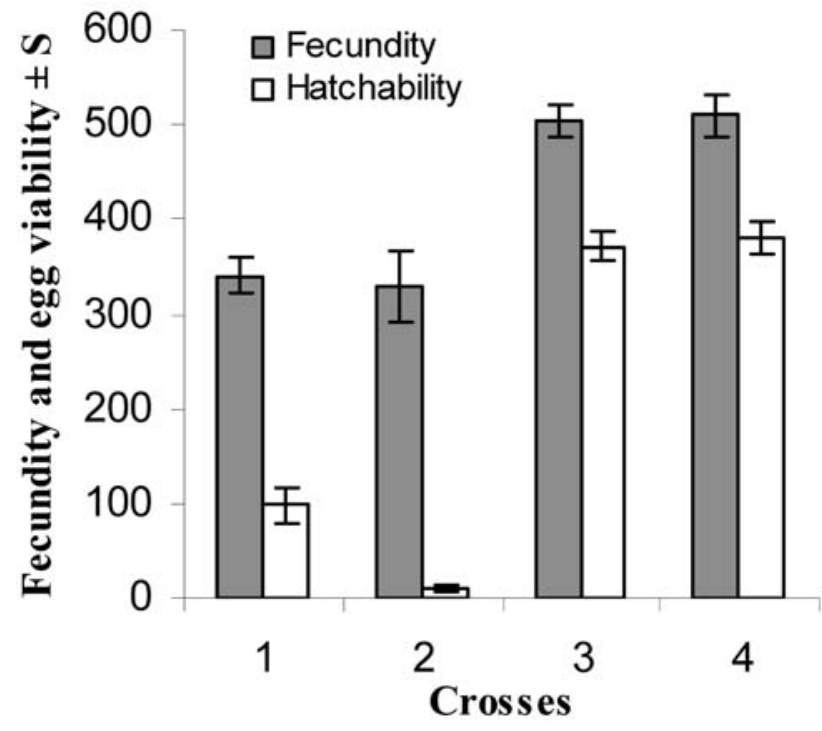

Fig. 6. Fecundity and egg viability (incompatibility) of four different crosses between uziflies: 1 - males $\times$ females (both from treated hosts); 2 - males (from untreated hosts) $\times$ females (from treated hosts); 3 - males $\times$ females (both from untreated hosts); 4 - males (from treated hosts) $\times$ females (from untreated hosts).

4.15 , d.f. $=13, \mathrm{p}<0.01)$ and the pupal weights were $7.804 \pm 0.054 \mathrm{gm}(\mathrm{n}=7)$ and $8.14 \pm 0.043 \mathrm{gm}(\mathrm{n}=8)$ for the untreated and treated larvae, respectively $(\mathrm{t}=5.06$, d.f. $=13, \mathrm{p}<0.01)$. Fig. 3 reveals that there was no corresponding significant increase in silk cocoon weight as it is $1.551 \pm 0.063 \mathrm{gm}$ for untreated larvae $(\mathrm{n}=7)$ and $1.526 \pm$ $0.0132 \mathrm{gm}$ for treated larvae $(\mathrm{n}=8)$ (the values are the means of 5 silk cocoons $\pm \mathrm{SE})(\mathrm{t}=0.61$, d.f. $=13, \mathrm{P}>$ 0.05). Further, Fig. 4 shows that the cocoon silk filament lengths were similar: $758.564 \pm 30.697 \mathrm{~m}(\mathrm{n}=10)$ for the untreated batch and $831.3 \pm 22.484 \mathrm{~m}(\mathrm{n}=15)$ for the tetracycline treated batches $(t=0.816$, d.f. $=23, p>0.05)$.

\section{Fecundity and fertility of treated silkmoths}

The fecundity of silkworms that had $0.1 \mathrm{mg} / \mathrm{ml}$ oxytetracycline added to their diet was significantly higher than that of untreated silkworms. In the crosses of untreated males $\times$ untreated females, treated males $\times$ untreated females, untreated males $\times$ treated females and treated males $\times$ treated females it was $395.83 \pm 10.370$, $(\mathrm{n}$ $=24), 417.783 \pm 11.56(\mathrm{n}=23), 550.464 \pm 8.855(\mathrm{n}=28)$ and $563.25 \pm 7.515(\mathrm{n}=16)$, respectively $(\mathrm{f}=8.18$, d.f. $=$ $87, \mathrm{p}<0.001)$. However, there were no significant differences in the percentages of the eggs that hatched in these crosses, which were $85.83 \%, 88.02 \%, 83.43 \%$ and $84.96 \%$, respectively. The average numbers of eggs that hatched are shown in Fig. 5.

\section{Fecundity and fertility of uziflies emerged from treated silkworms}

The fecundity of female uziflies that emerged from treated hosts was $340.875 \pm 19.94(\mathrm{n}=8)$ and $330.11 \pm$ $37.209(\mathrm{n}=9)$, which were significantly lower by $30-32 \%$ than the $510.2 \pm 16.9(\mathrm{n}=10)$ and $503.8 \pm$ $22.190(\mathrm{n}=10)$ of those that emerged from untreated hosts, $(f=99.50$, d. $f .=33, p<0.001)$. A similar trend was also observed in the number of eggs that hatched, which were $97.75 \pm 17.351,9.889 \pm 2.72,381.1 \pm 15.28$ and $372 \pm 17.215$, respectively (Fig. 6).

Apart from the effect on reproduction, the uziflies emerged from treated hosts one day earlier than those from untreated hosts.

\section{DISCUSSION AND CONCLUSION}

There have been several attempts, using physical, chemical and biological methods, to curtail the damage to silkworms caused by the uzifly, E. sorbillans, a serious tachinid endoparasitoid (Channabasavanna et al., 1993; Shivashankarappa \& Puttaraju, 1995). There is an obligate microorganism in the uzifly (Madhu \& Puttaraju, 2001; Puttaraju \& Madhu, 2002), which molecular genetic studies using $16 \mathrm{~S}$ rDNA and ftsZ gene analysis indicate that belongs to the genus Wolbachia (Chatterjee et al., 2003). In the present study, the antibiotic tetracycline was used to kill the Wolbachia in an attempt to minimise the infestation by the parasitoid and increase the yield of silk. The oral administration of tetracycline stimulated the growth and development of the silkworm, Bombyx mori L. (Fig. 1) and reduced the reproductive fitness of its parasitoid, the uzifly.

It is suggested that the antibiotic, as in the similar study by Venkatachalamurthy \& Sreenivasaya (1953), has a beneficial influence by controlling the intestinal flora of silkworm larvae, which may cause several diseases in silkworm. For example Spilarctia imparilis and Enterobacter sp. are pathogenic to silkworm (Govindan \& Devaiah, 1995), whereas silkworm infected with Escherichia coli produce minute amounts of antibacterial substances, which are beneficial to silkworm $(\mathrm{Qu}$ et al., 1984). Antibiotic treatment also decreased larval duration by a day and resulted in a significant increase in total cocoon weight including the pupa. However, the weight of the silk cocoon and silk filament length were not significantly affected. In addition to the increase in larval and cocoon weight, there was a definite and significant increase in pupal weight (Fig. 2), which was reflected in fecundity. This indicates that increased larval weight, cocoon weight, pupal weight and egg production are correlated, as was shown by Shyamala et al. (1960). This increased productivity may be due to the effect of tetracycline on food consumption, growth rate, conversion efficiency (Mukunda, 1999) and protein synthesis (Shyamala et al., 1960). Further, the absence of cytoplasmic incompatibility, sex ratio distortion and feminization of genetic males indicates the absence of Wolbachia in silkworm. PCR assay further confirms the absence of Wolbachia in silkworms, which is known to induce various types and levels of post-zygotic reproductive isolation in insects.

On the other hand, the uziflies that emerged from tetracycline-treated silkworms showed significant changes in reproductive behaviour. Of the eggs that resulted from crosses between male and female flies that emerged from treated silkworms 70 to $73 \%$ were infertile, 
indicating that Wolbachia may secrete proteins that are essential for uzifly reproduction/embryonic development. In addition, female uziflies that emerged from tetracycline treated silkworm laid 30\% fewer eggs indicating that Wolbachia effect is shifting facultative mode to obligate mode in this uzifly. This is not due to the toxicity of tetracycline on these uziflies as tetracycline has no effect on longevity, survival and behavior of the uziflies. The toxicity of tetracycline has also been excluded by a special experiment: direct antibiotics treatment for four generations was followed by rearing without antibiotics for three generations. The results of subsequent crossing (data not shown and unpublished) are compatible with the present experiment and indicate that the Wolbachia is evolving as one of the obligate mutualists in this uzifly parasite. These results accord with the data already published on Asobara tabida for which Wolbachia is essential for oogenesis (Dedeine et al., 2001, 2004). The reduced fecundity of the females that emerged from treated hosts may also be explained by a delay in egg production due to a delay in the uptake of nutrients due to the absence of Wolbachia. Interestingly, crosses between male flies that emerged from untreated hosts and female flies from treated hosts show clear cytoplasmic incompatibility. The cytoplasmic incompatibility is perhaps due to the combination of Wolbachia-infected sperm and uninfected eggs, which typically results in zygotic death (Werren, 1997). This can be best explained by first an "imprinting" effect on the male sperm, which occurs during maturation and before Wolbachia is excluded from the mature spermatozoan, and second a "rescue" effect, which occurs in Wolbachia-infected eggs and allows an imprinted sperm to successfully fertilize an infected egg (Werren, 1997). Sperm imprinting may be due to either Wolbachia proteins that modify the fly's chromosomes or the removal and/ or modification of host proteins that are necessary for the proper condensation and decondensation of the fly's chromosome set before and/or during zygote formation. Similarly, the presence of the same Wolbachia strain in the egg may result in the production and secretion of a rescue factor or in the recruitment of host molecules that are capable of rescuing the sperm "imprint" in a Wolbachia strain-specific manner. On the other hand, the crosses between male flies that emerged from tetracycline-treated silkworm and female flies from untreated hosts were partially sterile as $25-28 \%$ of the embryos died. Apart from the effect on reproduction, Wolbachia-infection delays the emergence of adult uziflies. The uziflies from treated hosts emerged one day earlier than those from untreated hosts, which indicate that the reduced fecundity could affect the rate of development. These results indicate that adding Wolbachiatargeted antibiotic tetracycline to the diet of silkworm has two benefits. Firstly, it increases silkworm growth and egg production and secondly, it decreases the reproductive fitness of the uzifly, Exorista sorbillans, by killing Wolbachia.

ACKNOWLEDGEMENTS. The authors are thankful to DBT New Delhi for financial assistance under grant No.
BT/PR2439/PBD/10/06/2001 and J. Milne of Mahidol University, Bangkok, Thailand for critical review of the manuscript.

\section{REFERENCES}

BARR A.R. 1980: Cytoplasmic incompatibility in natural populations of a mosquito, Culex pipiens L. Nature 283: 71-72.

BREEUWER J.A.J. 1997: Wolbachia and cytoplasmic incompatibility in the spider mite Tetranychus urticae and T. turkestani. Heredity 79: 41-47.

Breeuwer J.A.J., Stouthamer R., Burns D.A., Pelletier D.A., Weisburg W.G. \& Werren J.H. 1992: Phylogeny of cytoplasmic incompatibility microorganisms in the parasitoid wasp genus Nasonia (Hymenoptera: Pteromalidae) based on 16s ribosomal DNA sequences. Insect Mol. Biol. 1: 25-36.

Channabasavanna G.P., Siddappaji C. \& Dandin S.B. 1993: Integrated management of the Indian uzi fly, Exorista bombycis infesting the mulberry silkworm. In Channabasavanna G.P., Veeranna G. \& Dandin S.B. (eds): Recent Advances in Uzi Fly Research. Proceedings of the National Seminar on Uzi Fly and its Control, 16-17 January, 1992. Bangalore, India, pp. 227-237.

Chatterjee S.N., Mohandas T.P. \& Taraphdar T. 2003: Molecular characterization of the gene pool of Exorista sorbillans (Diptera: Tachinidae) a parasitoid of silkworm Bombyx mori, in India. Eur. J. Entomol. 100: 195-200.

Dedeine F., Vavre F., Fleury F., Loppin B., Hochberg M. \& Bouletreau M. 2001: Removing symbiotic Wolbachia bacteria specifically inhibits oogenesis in a parasitic wasp. Proc Natl Acad Sci. USA 98: 6247-6252.

Dedeine F., Vavre F., Fleury F., Shoemaker D.D. \& BouleTREAU M. 2004: Intra-individual coexistence of a Wolbachia strain required for host oogenesis with two strains inducing cytoplasmic incompatibnility in the wasp Asobara tabida. Evolution 58: 2167-2174.

Govindan R. \& Devaiah M.C. 1995: Bacterial Flacherie of Silkworm. Communication center UAS, Bangalore.

Hoerauf A., Nissen-Pahle K., Schmetz C., Henkle-Duhrsen K., Blaxter M.L., Buttner D.W., Gallin M.Y., Al-Qaoud K.M., Lucius R. \& Fleischer B. 1999: Tetracycline therapy targets intracellular bacteria in the filarial nematode Litomosoides sigmodontis and results in filarial infertility. J. Clin. Invest. 103: 11-18.

Hurst G.D.D., Jiggins F.M., Schulenburg J.H.G.V.D., Bertrand D., West S.A., Goriacheva I.I., Zakharov I.A., Werren J.H., Stouthamer R. \& Majerus M.E.N. 1999: Malekilling Wolbachia in two species of insect. Proc. R. Soc. Lond. (B) 266: 735-740.

JEYAPRAKASH A. \& Hoy M.A. 2000: Long PCR improves Wolbachia DNA amplification: WSP sequence found in $76 \%$ of sixty-three arthropod species. Insect Mol. Biol. 9: 393-405.

KambHampati S., Rai K.S. \& BuRGun S.J. 1993: Unidirectional Cytoplasmic Incompatibility in the mosquito, Aedes albopictus. Evolution 47: 673-677.

Khan I.A. \& Khanum A. 1994: Fundamentals of Biostatistics. Ukaaz Publications, Hyderabad, India, pp. 392-431.

KozeK W.J. 1997: Transovarially-transmitted intracellular microorganisms in adult and larval stage of Brugia malayi. $J$. Parasitol. 63: 992-1000.

Langworthy N.G., Renz A., Mackensted U., Henkle-Duhrsen K., Bronsvoort M.B., Tanya V.N., Donnelly M.J. \& Trees A.J. 2000: Macro filarial activity of tetracycline against the filarial nematode Onchocerca ochengi: elimination of Wolbachia species precedes worm death and suggests a dependent relationship. Proc. R. Soc. Lond. (B) 367: 1063-1069. 
Madhu M. \& Puttaraju H.P. 2001: Cytological and molecular incidence for Wolbachia infection in Uzi flies of Exorista species. Cytologia 66: 197-203.

Mahadevappa D., Halliyal V.G., Shankar D.G \& Bhandiwad R. 2000: Mulberry Silk Reeling Technology. Oxford and IBH publication Co., India.

Manjunatha H.B. \& Puttaraju H.P. 1993: The egg of the uzifly, Exorista sorbillans (Diptera: Tachinidae). Appl. Entomol. Zool. 28: 574-577.

Min K.T. \& Benzer S. 1997: Wolbachia, normally a symbiont of Drosophila, can be virulent, causing degeneration and early death. Proc. Natl. Acad. Sci. USA 94: 10792-10796.

MuKunda N. 1999: Effect of Application of Tetracycline and Sulphamethaxazole on the Growth and Development of Silkworm Bombyx mori. M.Sc. Dissertation, Bangalore University, Bangalore.

O’Neill S.L., Giordano R., Colbert A.M.E., Karr T.L. \& RobERTSON H.M. 1992: 16s rDNA phylogenetic analysis of the bacterial endosymbionts associated with cytoplasmic incompatibility in insets. Proc. Natl. Acad. Sci. USA 89: 2699-2702.

Puttaraju H.P. \& Madhu M. 2002: Presence of Wolbachia endosymbionts in different silkworm species and races and in their Uzi fly parasites. J. Invertebr. Pathol. 79: 120-122.

Qu X.M., Qi G.R. \& HuANG Z.R. 1984: Comparative studies on antibacterial substances of silkworm after Escherichia coli infection and ultrasonic treatment. Acta Entomol. Sin. 27: 275-279.

Rousset F., Bouchon D., Pintureau B., Juchault P. \& Solignac M. 1992: Wolbachia endosymbionts responsible for various alterations of sexuality in arthropods. Proc. R. Soc. Lond. (B) 250: $91-98$.

SAmbrook J., Fritsch E.F. \& Miniatis T. 1989: Molecular Cloning: A Laboratory Manual. 2nd ed. Cold Spring Harbor Laboratory Press, Cold Spring Harbor, New York, 999 pp.

Shivashankarappa L.H. \& Puttaraju H.P. 1995: Effect of diflubenzuron on the reproduction of uzifly, Exorista bombycis (E. sorbillans) (Diptera: Tachinidae). Sericologia 15: 763-766.
Shyamala M.B., Sharada K., Bhat M.G. \& Bhat J.V. 1960: Chloromycetin in the nutrition of the silkworm Bombyx mori L. II Influence on digestion and utilization of protein, fat and minerals. J. Insect Physiol. 4: 229-234.

Stouthamer R., Breeuwer J.A.J., Luck R.F. \& Werren H. 1993: Molecular identification of microorganisms associated with parthenogenesis. Nature 361: 66-68.

TAYlor M.J. \& HoERauf A. 2001: A new approach to the treatment of filariasis. Car. Opin. Infect. Dis. 14: 727-731.

Trpis M., Perrone J.B., Reissig M. \& Parker K.L. 1981: Control of cytoplasmic incompatibility in the Aedes scuttellaris complex. J. Heredity 72: 313-317.

Ullal S.R. \& Narasimhanna M.N. 1994: Handbook of Practical Sericulture. Arun Ramanath, Omkar offset, Bangalore.

Vala F., Van opinnen T., Breeuwer J.A. \& Sabelis M.W. 2003: Genetic conflict over sex-ratio: mite-endosymbiont interactions. Am. Nat. 161: 254-266.

Venkatachalamurthy M. R. \& Sreenivasaya M. 1953: Effect of antibiotics on the growth of the silkworm, Bombyx mori L. Nature 173: 684-685.

WADE M.J. \& ChANG N.W. 1995: Increased male fertility in Tribolium confusum beetle after infection with the intracellular parasite Wolbachia. Nature 51: 72-74.

WERREN J.H. 1997: Biology of Wolbachia. Annu. Rev. Entomol. 42: 587-609.

WerRen J.H. \& Beukeboom L. 1998: Sex determination, sex ratios and genetic conflict. Annu. Rev. Ecol. Syst. 29: 233-261.

WerRen J.H., Windsor D. \& Guo L. 1995: Distribution of Wolbachia among neotropical arthropods. Proc. R. Soc. Lond. (B) 262: 197-204.

Zabalou S., Riegler M., Theodorakopoulou M., Stauffer C., SAVAKIS C. \& BourtzIS K. 2004: Wolbachia-induced cytoplasmic incompatibility as a means for insect pest population control. Proc. Natl. Acad. Sci. USA 101: 15042-15045.

Received June 7, 2004; revised and accepted April 25, 2005 